\title{
Influence of dispersion slope on soliton spectral tunneling in photonic crystal fiber
}

\author{
YunXia YANG ${ }^{1}$, Hua YANG ${ }^{1,2^{*}}$, Xiongfeng TONG ${ }^{3}$, Saili ZHAO ${ }^{1}$, Shuyuan CHEN $^{1}$ \\ ${ }^{1}$ College of Information Science and Engineering, \\ Key Laboratory for Micro/Nano Optoelectronic Devices of Ministry of Education, \\ Hunan University, Changsha 410082, P.R. China \\ ${ }^{2}$ Synergetic Innovation Center for Quantum Effects and Application, Hunan Normal University, \\ Changsha 410082, P.R. China \\ ${ }^{3}$ China Unicom Changsha Branch, \\ Changsha 410082, P.R. China \\ ${ }^{*}$ Corresponding author: huayang@hnu.edu.cn
}

\begin{abstract}
We report a numerical investigation of how the dispersion slope affects the soliton spectral tunneling (SST) in a photonic crystal fiber with three zero dispersion wavelengths. It is discovered that a larger dispersion slope makes group-velocity mismatch between the initial soliton and the transferred wave thereby suppressing the SST effect, while a proper decrease of the dispersion slope enhances the SST effect to widen a supercontinuum range. Besides, we find a soliton-like leaking dispersion wave, which can sustain information and energy for a short time within a particular spectral range.
\end{abstract}

Keywords: soliton spectral tunneling, supercontinuum generation, dispersion slope.

\section{Introduction}

The generation of ultra-broadband supercontinuum (SC) has been a hot topic as its favorable implications in photochemistry and spectroscopy, etc. [1, 2]. The dynamics of a dispersive wave (DW) and optical soliton play an essential role in the process of $\mathrm{SC}$ generation. The DW originates from the femtosecond soliton pulse perturbed by higher-order effects and is critical for controlling the shorter wavelength edge of the spectrum [ $\underline{3}-\underline{5}]$. However, the spectrum at the longer wavelength edge is controlled by the Raman-induced soliton self-frequency shift (SSFS), which produces a continuous redshift with respect to the center wavelength of the soliton pulse $[\underline{4}, \underline{5}]$. Ordinarily, optical solitons exist only in anomalous group velocity dispersion (GVD) regimes [ $[\underline{6}, \underline{7}]$. 
However, theoretical studies have demonstrated that the Raman-induced SSFS allows a soliton to tunnel through a spectrally limited regime of normal dispersion sandwiched by two anomalous GVD regimes, and couple its energy to the latter anomalous GVD regime thereby forming a new soliton $[\underline{8}, \underline{9}]$. Because of many similarities between the above phenomenon and quantum mechanical tunneling across a potential barrier, this phenomenon is named soliton spectral tunneling (SST) according to the evolution phenomena in the spectral domain [10-12].

The generation process of the SST effect is complicated. It demands a potential barrier in the dispersion profile, which is a normal GVD regime surrounded by two anomalous ones. Such characteristics is challenging to be satisfied in traditional fibers; however, it is realizable in PCFs due to controllable dispersion characteristics of PCF $[13,14]$. By adjusting the geometric structure of PCF, we can obtain fibers with multiple zero dispersion wavelengths (ZDWs) of different dispersion profiles in practice [15]. Fundamentally, the SST effect is primarily motivated by the Raman-induced SSFS to constantly transfer energy to the DW when the soliton propagates through an optical fiber [12]. Accordingly, the phase-match condition between the initial soliton and the transferred wave is another requirement for the SST effect $[10,12]$. If the transferred wave is group velocity (GV) matched to the initial soliton, which satisfies the condition of soliton phase matching (PM), then the transferred wave can form a tunneled soliton due to the delicate equilibrium between nonlinear self-compression and dispersive broadening. Otherwise, the transferred wave performs as a linear DW with leakage structure. Thus, the SST effect is also considered to be a sort of DW generation induced by a soliton $[\underline{16}, \underline{17}]$.

Since 1978, when NEwELL first predicted that the tunneling effect exists in nonlinear media [18], a considerable effort has been put into studies about SST controlled by the nonlinear Schrödinger equation (NLSE) [10, 19-21]. For the observation of SST, a few index-guiding holey fibers with a tunable potential barrier have been designed by POLETTI et al. [10]. ZhaO et al. [21] numerically studied the influence of a chirp on the SST in the process of supercontinuum. However, there are as yet little studies focusing on how the magnitude of the dispersion slope impacts on the process of SST in a fiber. One of the dispersion slope's primary effects on ultra-short pulses is that the pulse peak shift varies linearly with the transmission distance and the lead or delay of the pulse is determined by the sign of the dispersion slope of the fiber [22]. There have been some studies indicating that the dispersion slope has an impact on both DWs and fundamental solitons in SC generation [23, 24]. For example, Joly et al. [23] have shown the nonlinear behavior of solid-core PCFs near a negative-slope ZDW. Also, YANG et al. [24] have numerically demonstrated DW generation in PCFs, including both negative and positive dispersion slopes for a pump wavelength. In the current work, we discuss the impact of the dispersion slope on the SST effect in a PCF with three ZDWs. The interaction between Raman solitons and DWs is a vital influence factor in the process of SST. Thus, analyzing the evolution of the SST when exploring the effect of the dispersion slope will be of great significance. It may inspire a new way to harness SST for SC generation. 
This article is organized as follows. A basic theoretical model for the input pulse propagating in a fiber that considers high-order dispersion and higher-order nonlinear effects is depicted in Section 2. In Section 3, the numerical simulations concerning the impact of different dispersion slopes on the SST are then discussed in detail. Finally, we summarize our numerical simulation results in brief in Section 4.

\section{Numerical model}

For the numerical model of ultra-short pulse propagation in a PCF with three ZDWs, a well-known and universally used NLSE can be described as

$$
\begin{aligned}
\frac{\partial A(z, T)}{\partial z}= & \sum_{k \geq 2} \frac{i^{k+1} \beta_{k}}{k !} \frac{\partial^{k} A}{\partial T^{k}} \\
& +i \gamma\left(1+\frac{i}{\omega_{0}} \frac{\partial}{\partial T}\right)\left[A(z, T) \int_{-\infty}^{+\infty} R\left(T^{\prime}\right)\left|A\left(z, T-T^{\prime}\right)\right|^{2} \mathrm{~d} T^{\prime}\right]
\end{aligned}
$$

The NLSE has been described in detail elsewhere [15, 25], but here we consider a brief description in pursuit of completeness. In Eq. (1), $A(z, T)$ is the complex amplitude of the time-domain pulse envelope; $T$ and $z$ are, respectively, the time parameter and dimensional propagation distance at the group velocity of the input pulse; $\gamma$ refers to the nonlinear parameter of the optical fiber; $\beta_{k}$ denotes the dispersion coefficient of $k$-th order at the pump carrier frequency $\omega_{0}$, and $R(T)$ is the nonlinear response function $[\underline{1}, \underline{15}]$ :

$$
R(T)=\left(1-f_{\mathrm{R}}\right) \delta(T)+f_{\mathrm{R}} h_{\mathrm{R}}(T)
$$

which includes Raman and Kerr components. The order of dispersion parameter is up to 10 to reach a good dispersion profile of PCFs. Meanwhile, the fiber loss is neglected to obtain a physical understanding of the effects, as only a short fiber length is considered in the simulations. The injected pulse we adopted is the non-chirped hyperbolic secant pulses in the numerical simulation:

$$
A(0, T)=\sqrt{P_{0} \operatorname{sech}\left(T / T_{0}\right)}
$$

The behavior of SST is strongly influenced by the incident parameters, thus making it possible to manipulate the SST process by adjusting the initial conditions. At the same time, a PCF is chosen as the appropriate transmission medium owing to its controllable nonlinear and dispersion features [16]. The dispersion profile and relative group delay of the optical fiber, considered here as a function of the wavelength, are depicted in Fig. 1. The adopted PCFs have three ZDWs $\left(\lambda_{1}<\lambda_{2}<\lambda_{3}\right)$ located respectively at $\sim 797,922$ and $1016 \mathrm{~nm}$, which split the entire spectrum into four regimes (short wavelength normal GVD regime $\left(\mathrm{N}_{1}: \lambda \leq \lambda_{1}\right)$, short wavelength anomalous GVD re- 


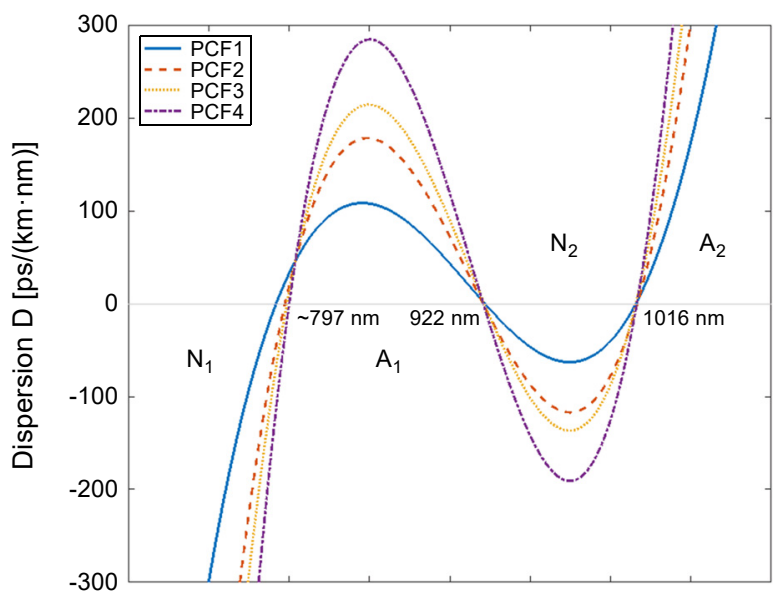

a

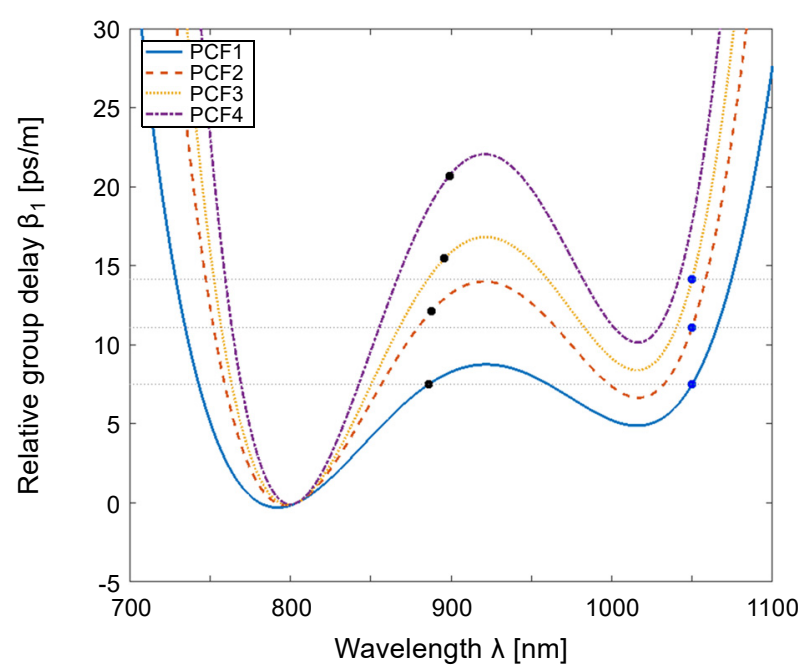

b

Fig. 1. The curve of dispersion parameter (a) and relative group delay (b) of PCF1 (blue solid curve), PCF2 (orange dashed curve), PCF3 (yellow dotted curve) as well as PCF4 (purple dotted-dashed curve).

gime $\left(\mathrm{A}_{1}: \lambda_{1} \leq \lambda \leq \lambda_{2}\right)$, long wavelength normal GVD regime $\left(\mathrm{N}_{2}: \lambda_{2} \leq \lambda \leq \lambda_{3}\right)$, and long wavelength anomalous GVD regime $\left(\mathrm{A}_{2}: \lambda>\lambda_{3}\right)$ ), as shown in Fig. 1a. This situation is ideal for researching the SST effect as two anomalous GVD regimes surround the normal GVD regime. There are four PCFs, namely PCF1 to PCF4, which are successively adjusted by increasing the dispersion slope. They are obtained through a proper choice of higher-order dispersion coefficients and guaranteeing consistent $\beta_{2}$, which ensure that only the dispersion slope is changed while keeping the soliton order constant. Use $\beta_{3}$ to indicate the difference in dispersion slope, setting the value as $\beta_{3.1}=0.3945 \mathrm{ps}^{3} \mathrm{~km}^{-1}$, $\beta_{3.2}=0.7734 \mathrm{ps}^{3} \mathrm{~km}^{-1}, \beta_{3.3}=0.9624 \mathrm{ps}^{3} \mathrm{~km}^{-1}$, and $\beta_{3.4}=1.3411 \mathrm{ps}^{3} \mathrm{~km}^{-1}$. For the sake of scientific rigor, we keep the consistent nonlinear coefficient of $\gamma=0.146 \mathrm{~W}^{-1} \mathrm{~m}^{-1}$ by properly adjusting the fiber parameters and uniformly use a pulse of width $T_{0}=90 \mathrm{fs}$ of 
wavelength $\lambda_{0}=804 \mathrm{~nm}$. Furthermore, we set the peak power at $1 \mathrm{~kW}$ and consider a fiber length of $0.45 \mathrm{~m}$.

\section{Numerical results and discussions}

To fully comprehend the process of SST, we firstly limit our observations to the output spectrogram traces of a successful soliton tunneling by using X-FROG technology, including temporal (bottom axis) and spectral (left axis) content of the signal, see Fig. 2. The numerical X-FROG patterns are presented at fiber lengths of $0.001,0.05,0.08$,
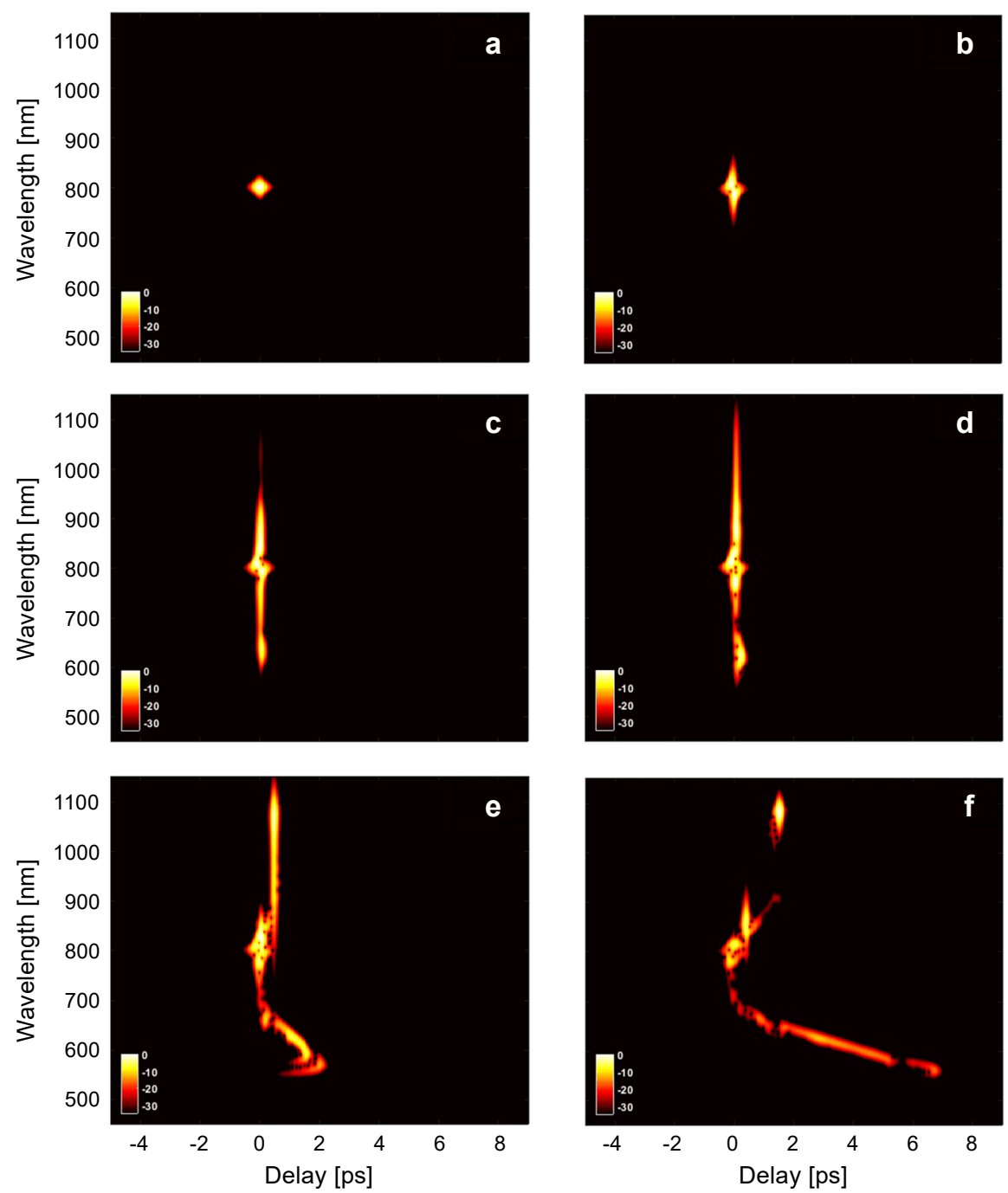

Fig. 2. The numerical X-FROG patterns of the input pulse for different propagation lengths of $0.001 \mathrm{~m} \mathrm{(a),}$ $0.05 \mathrm{~m}(\mathbf{b}), 0.08 \mathrm{~m}(\mathbf{c}), 0.1 \mathrm{~m}(\mathbf{d}), 0.2 \mathrm{~m}(\mathbf{e})$, and $0.4 \mathrm{~m}(\mathbf{f})$. 
$0.1,0.2$ and $0.4 \mathrm{~m}$, respectively. Figure 2 a shows that the pulse width of the pump pulse is extremely narrow at the input of a PCF. In the initial stage, the spectrum of femtosecond laser pulses is symmetrically broadened due to SPM, see Fig. 2b. Typically, optical pulses transmit in the form of high-order solitons and are susceptible to HOD as well as other nonlinear effects, such as four-wave mixing (FWM) and stimulated Raman scattering (SRS). After strong temporal compression, the ejected pulse achieves maximum bandwidth, after which perturbations of the linear and nonlinear effects initiate the input pulse breakup. Consequently, a series of fundamental solitons with diverse peak powers and durations are injected. Soliton split enables parts of energy to be transferred to the narrow-band resonance of the normal GVD regime, and thereby emit DWs when meeting the PM condition of the dispersion wave. Figure $2 \mathbf{c}$ shows that B-DW is generated at a shorter wavelength normal GVD regime. Then the fundamental soliton (i.e., the initial soliton) experiences redshift resulting from the Raman response. When the initial soliton reaches to the second ZDW, the generation of R-DW at longer wavelength normal GVD regime means that the energy channel of tunneling is formed, see Fig. 2d. If the transferred wave $(1050 \mathrm{~nm})$ is $\mathrm{GV}$-matched to the initial soliton $(861 \mathrm{~nm})$ and the soliton PM condition is fulfilled, the energy of the initial soliton will be effectively transformed to the transferred wave through the channel, as shown in Fig. 2e.
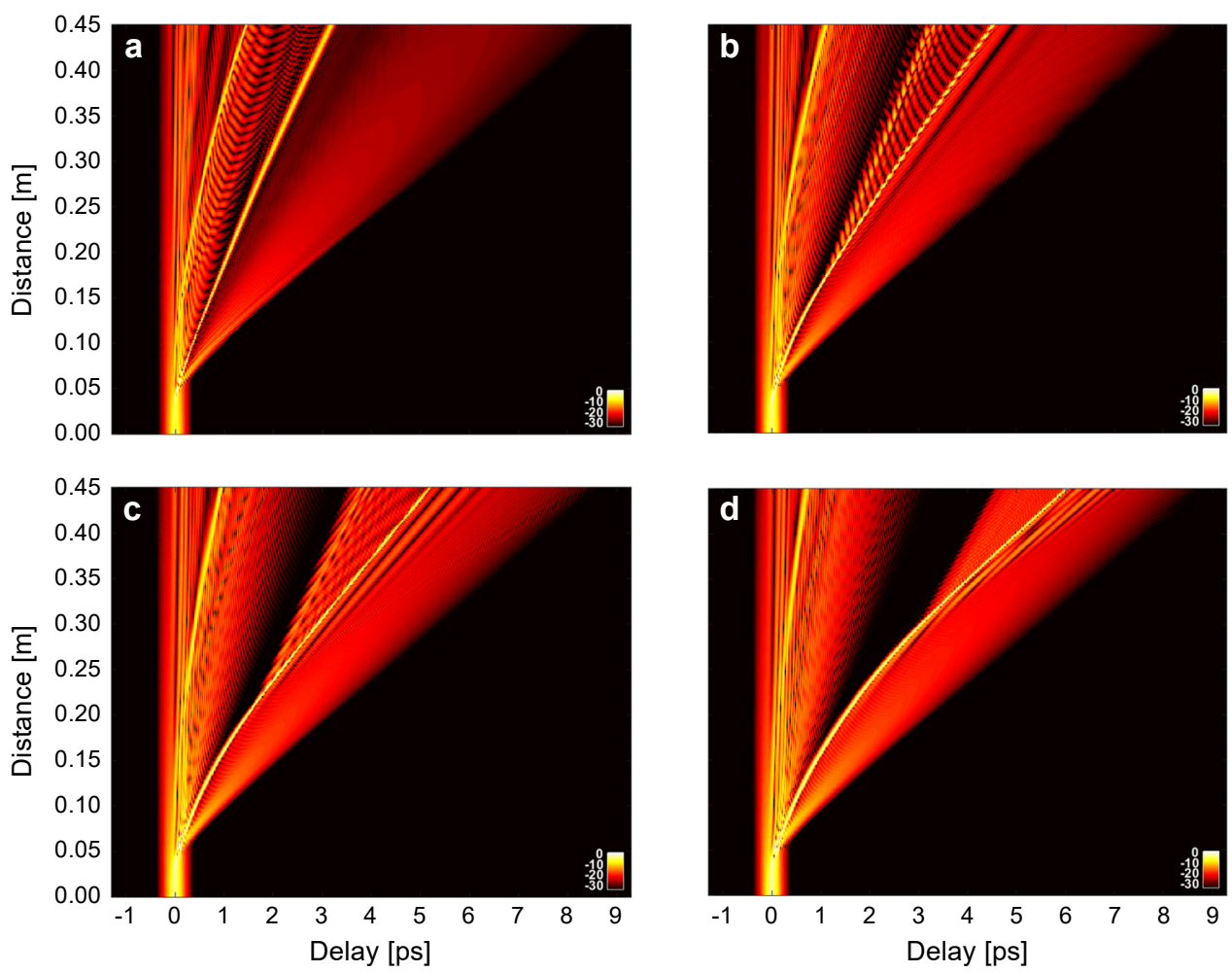

Fig. 3. The corresponding temporal evolution as a function of fiber length under the condition of different dispersion slopes in the PCF1 (a), PCF2 (b), PCF3 (c), and PCF4 (d). 
Energy coupling from the initial soliton and R-DW continuously occurs until it is fully coupled into the transferred wave to form a soliton state, namely, tunneled soliton. Figure $2 \mathbf{f}$ shows the coupling is completed and the initial soliton as well as R-DW disappear, which is a perfect soliton tunneling.

In order to get a comprehensive understanding of the impacts of the dispersion slope on SST, let us start with the corresponding temporal evolution under the condition of different dispersion slopes respectively in PCF1 to PCF4 with $0.45 \mathrm{~m}$ length, corresponding to Figs. 3a-3d. From the dispersion parameter curve of Fig. 1a, it can be found that the dispersion slope near the first ZDW is positive, while the dispersion slope near the second ZDW is negative. Therefore, according to the phase matching condition of the dispersion wave, B-DW is generated at the back edge of the first soliton (i.e., initial soliton) and R-DW is generated at the front. We discover that the alteration of a dispersion slope value has a significant effect on the generation of DW, thus leading to changes in the energy distribution of DWs. On the other hand, changing the dispersion slope value also affects the velocity of the soliton. It can be seen that transmittal velocities of the first soliton gradually decrease while the related delays become higher when increasing the dispersion slope.

Figure 4 presents the spectral evolution and related output spectra with different dispersion slope values. As soon as the pump is launched into a PCF, the pump spectrum broadens purely through SPM up to $\sim 0.04 \mathrm{~m}$. Then a train of fundamental solitons appears subsequently, along with the radiation of the related DWs. In Fig. 4a, it can be observed that B-DW generates at a distance of $\sim 0.05 \mathrm{~m}$ in $\mathrm{N}_{1}$. The first splitting fundamental soliton (i.e., initial soliton) undergoes Raman-induced SSFS and continues to redshift. When it reaches the second ZDW at $\sim 0.06 \mathrm{~m}, \mathrm{R}-\mathrm{DW}$ radiates in $\mathrm{N}_{2}$ and forms an energy channel, which couples the energy of the initial soliton to $A_{2}$. At this time, let us see filled circles with black or blue colors in Fig. 1b respectively which correspond to the $\beta_{1}$ of the initial soliton and transferred wave at the corresponding wavelength under different conditions. In such conditions, there is little difference between the $\beta_{1}$ of the two circles in PCF1, which suggests the GV-matching condition is satisfied between them and thereby the initial soliton will continue to deliver energy to the transferred wave until the coupling is completed at $\sim 0.2 \mathrm{~m}$. The transferred wave becomes a tunneled soliton in $\mathrm{A}_{2}$, and the R-DW disappears. When increasing the dispersion slope, spectral evolution makes a huge difference, as Fig. 4b illustrates. As can be seen from the temporal evolution, the initial soliton moves more slowly, which results in the suppression of SSFS. Consequently, it needs to transmit a longer distance $(\sim 0.18 \mathrm{~m})$ to reach the second ZDW. In Fig. 1b, two circles in PCF2 have quite different $\beta_{1}$, which indicates GV-mismatching. At this time, the transferred wave becomes a leakage-like DW, while the generated R-DW does not disappear but continues to propagate. The energy of the initial soliton is not effectively coupled, and the residual energy remains near the second ZDW, so the SST fails. In addition, because the suppression of SSFS leads to the phase change of the initial soliton, the location of B-DW generation is changed to longer wavelength. Accordingly, the spectrum becomes narrower. However, as the dispersion slope continues to increase, the trend of phenomenal change 

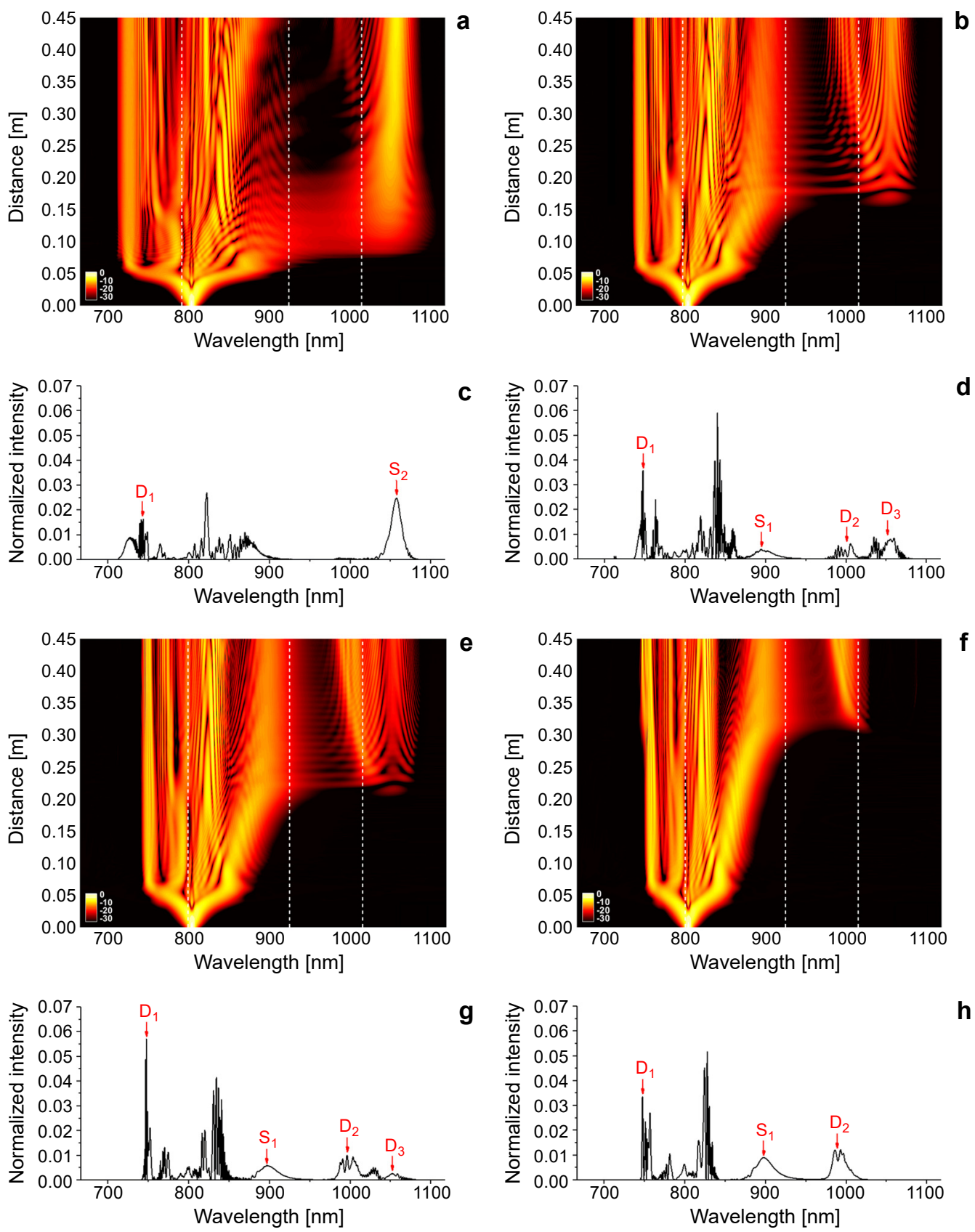

Fig. 4. Spectral evolution as a function of fiber length $(\mathbf{a}, \mathbf{b}, \mathbf{e}, \mathbf{f})$ and the corresponding output spectra $(\mathbf{c}, \mathbf{d}, \mathbf{g}, \mathbf{h})$ under the condition of different dispersion slope values: adjusted by the increasing dispersion slope, corresponding to PCF1 (a, c), PCF2 (b, d), PCF3 (e, g) and PCF4 (f, h). The vertical dashed lines imply the three ZDWs of the optical fiber, separating domains $\mathrm{N}_{1}, \mathrm{~A}_{1}, \mathrm{~N}_{2}$, and $\mathrm{A}_{2}$, in which the normal GVD regime $\mathrm{N}_{2}$ is surrounded by two anomalous ones: $\mathrm{A}_{1}$ and $\mathrm{A}_{2} . \mathrm{D}_{1}, \mathrm{D}_{2}, \mathrm{D}_{3}, \mathrm{~S}_{1}$, and $\mathrm{S}_{2}$ respectively stand for the various components of the output spectra: B-DW, R-DW, leakage-like DW, initial soliton and tunneled soliton. 
is further reflected in Fig. 4e. The distance of the initial soliton reaching to the second $\mathrm{ZDW}$ increases to $\sim 0.22 \mathrm{~m}$. GV-mismatching is more serious because the difference of relative group delay is greater, as seen from the value of two circles in PCF3 of Fig. $1 \mathbf{b}$. The energy of the generated leakage-like DW is weakened, the R-DW energy is enhanced, the residual energy of the initial soliton increases and the spectrum width is narrower. Such phenomena are more distinct in Fig. $4 \mathbf{f}$. The initial soliton propagates near the second ZDW due to the spectral recoil effect, and the leakage-like DW disappears completely, so SST does not exist. At this point, the soliton slows down dramatically, overlapping with the radiated B-DW in the temporal domain, and the soliton trapping of DWs is produced by FWM. The trapped B-DW is generated at a transmission distance of $\sim 0.28 \mathrm{~m}$.

The energy of each component at the output also varies significantly, as sketched in Figs. $4 \mathbf{c}, 4 \mathbf{d}, 4 \mathbf{g}, 4 \mathbf{h}$. Firstly, it is obvious in Fig. $4 \mathbf{c}$ that the tunneled soliton (labeled $\mathrm{S}_{2}$ ) is formed and R-DW almost does not exist, which is a typical SST. When the dispersion slope is increased in Fig. 4d, the tunneled soliton disappears due to GV-mismatching, and the leakage-like DW (labeled $\mathrm{D}_{3}$ ) with weak energy is formed. R-DW (labeled $\mathrm{D}_{2}$ ) always exists in the whole transmission process without establishing an effective channel. Some energy of the initial soliton (labeled $\mathrm{S}_{1}$ ) remains near the second ZDW. Consequently, SST fails. Meanwhile, the intensity of B-DW (labeled $\mathrm{D}_{1}$ ) has a bigger amplitude because of the narrowed spectrum but constant total energy. With the increase of the dispersion slope, the trend of energy change in each part is more prominent in Fig. 4g. As the dispersion slope continues to increase in Fig. 4h, the severe GV-mismatching causes the leakage-like DW to disappear completely and SST does not exist. The initial soliton energy cannot be coupled to $\mathrm{A}_{2}$, so its energy increases and radiating is stronger in R-DW. Part of B-DW energy is transferred to the trapped DW, which reduces its amplitude.

Figure 5 shows the output spectrogram trajectories of different dispersion slope values. First, we observe the change of the transferred wave. In Figs. 4a, 4c, due to GV-matching, the energy of initial soliton and R-DW is almost all coupled into the transferred wave, and the transferred wave becomes a distinct tunneled soliton. While in Figs. $4 \mathbf{b}, 4 \mathbf{d}$ and $4 \mathbf{e}, 4 \mathrm{~g}$, the initial soliton energy cannot be effectively coupled because of GV-mismatching, which gives rise to the formation of soliton-like leakage in $\mathrm{A}_{2}$. The leakage initially forms a soliton-like shape but gradually dissipates as propagating along the optical fiber. So it is a leaking DW instead of a soliton. Figures $4 \mathbf{f}, 4 \mathbf{h}$ reveals that the initial soliton ceases to redshift around the second ZDW because of spectral recoiling from the amplification of R-DW, which leads to the absence of leakage -like DW and, accordingly, the SST. Besides, we find that the relative group delay of the initial soliton at the output increases with a larger dispersion slope value, leading to slowing down and phase changing of the soliton. Therefore, when meeting the PM condition of the dispersion wave, the redshift of corresponding B-DW narrows the spectrum at a shorter wavelength. And the suppression of the SST also causes the spectrum at the longer wavelength to be narrowed. It is concluded that an increase in the dispersion slope suppresses the SSFS, which results in the occurrence of tunneling at a longer 

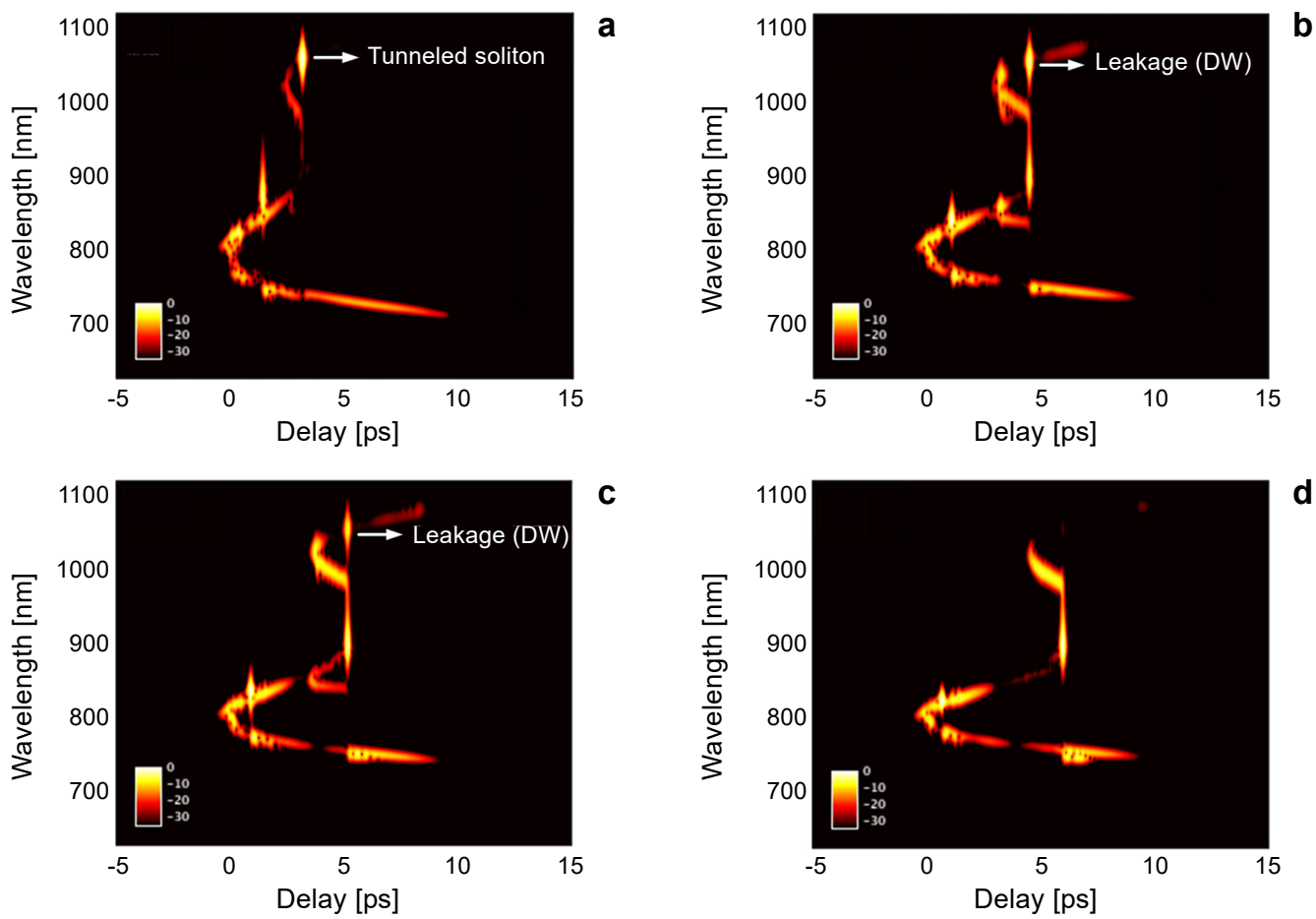

Fig. 5. The numerical X-FROG patterns with different dispersion slope values at the output of PCF1 (a), PCF2 (b), PCF3 (c), and PCF4 (d) with three ZDWs.

transmission distance. Moreover, increasing dispersion slope makes GV difficult to match, which is not conducive to SST effect, and narrows the spectrum finally.

In Fig. 6, we show the effect of a dispersion slope value on the energy conversion rate of B-DW and tunneled soliton in case of successful tunneling of a soliton. Note that, in SST, if the dispersion slope value is lower than its threshold, the soliton will move too fast to observe its tunneling. The threshold of successful tunneling, which is the result of our simulation experiment, is $\sim 0.4 \mathrm{ps}^{3} \mathrm{~km}^{-1}$. The dispersion slopes of case I and case II are respectively $\beta_{3 . \mathrm{I}}=0.5128 \mathrm{ps}^{3} \mathrm{~km}^{-1}$ and $\beta_{3 . \mathrm{II}}=0.6076 \mathrm{ps}^{3} \mathrm{~km}^{-1}$. The transfer ratio of power can be formulated by $\eta=E_{\text {part }} / E_{\text {total }}$ (where $E_{\text {part }}$ represents the energy of the corresponding part, and $E_{\text {total }}$ is the total energy of the optical pulse). It reveals that the variation of a dispersion slope has little effect on the final B-DW energy ratio ( 0.05), as shown in Fig. 6a, which can extend to the failed SST condition and is in accordance with the previous explanation. By contrast, the energy ratio of tunneled solitons is obviously influenced by the dispersion slope in Fig. $6 \mathbf{b}$. The tunneled solitons of three conditions are gradually formed one by one, and their energy ratios begin to rise at different rates. The energy ratio of PCF 1 takes the lead in achieving its peak value of $\sim 0.245$ at about $0.3 \mathrm{~m}$ and remains invariable. In case I, the energy ratio of tunneled soliton reaches a stable value of $\sim 0.23$ at a longer transmission distance of about $0.35 \mathrm{~m}$. While the energy ratio of the tunneled soliton in case II reaches 


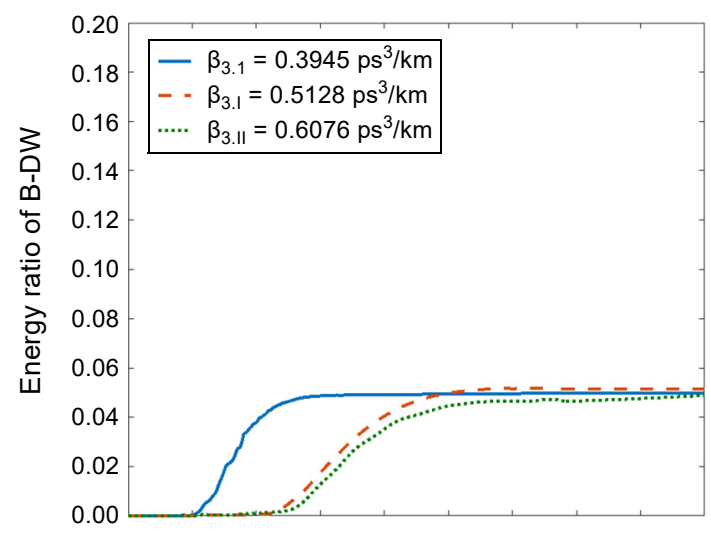

a

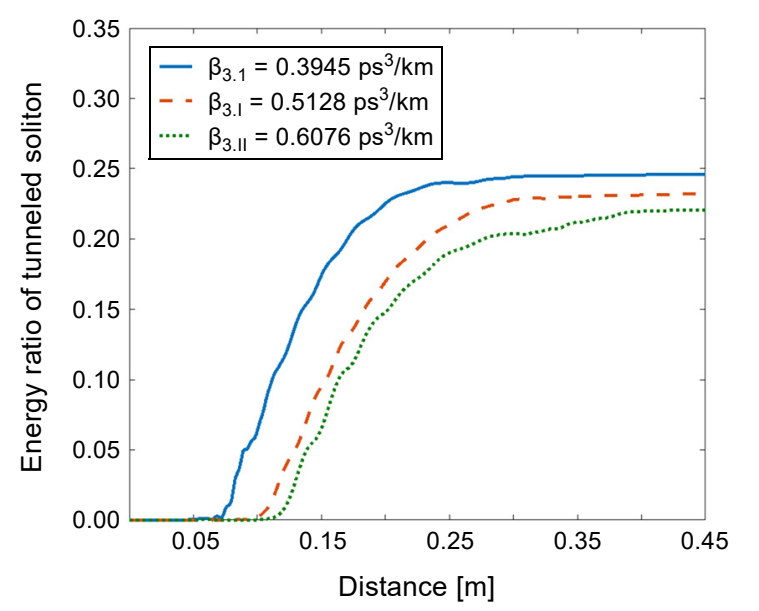

b

Fig. 6. The evolution in energy ratio of B-DWs (a) and tunneled solitons (b) in PCF1 (blue solid curve), case I (orange dashed curve), and case II (green dotted curve) as a function of propagation distance.

a peak of $\sim 0.22$ at about $0.39 \mathrm{~m}$ and keeps unchanged. We can conclude that the dispersion slope does not affect the final energy ratio of B-DW, while, on the premise of successful tunneling of a soliton, the energy ratio of the tunneled soliton is improved and conversion rate is faster with the decrease of the dispersion slope value.

\section{Conclusion}

In this paper, we have numerically demonstrated the impact of the dispersion slope on SST during SC generation in different PCFs with three ZDWs. As the dispersion slope increased, SST appears at a farther transmission distance owing to the delay of Raman -induced SSFS. In this case, a larger dispersion slope causes the curve of relative group delay to fluctuate more intensely, which makes it difficult to achieve the GV-matching between the initial soliton and the transferred wave, thus suppressing the occurrence of SST effect. In contrast, a proper decrease of the dispersion slope (but over the threshold 
to ensure SST successfully) causes the enhancement of SSFS and facilitates meeting the GV matching condition. Under the conditions, the tunneled soliton has a higher energy ratio, thereby strengthening the SST process to make SC with a much broader spectral range. Therefore, the dispersion slope plays a significant role in the process of SST, which offers a novel approach to acquire wider SC. Moreover, we get a soliton -like leaking DW when adjusting the dispersion slope appropriately. The leakage may carry information and energy at first and dissipates shortly after.

Acknowledgments - This study was supported by the National Natural Science Foundation of China (grant number 61275137), the Natural Science Foundation of Hunan Province of China (grant number 2018JJ2061) and the Key Research and Development Program of Changsha City (grant number kq1901027).

\section{References}

[1] Hosseini P., Ermolov A., Tani F., Novoa D., Russell P.St.J., UV soliton dynamics and Raman-enhanced supercontinuum generation in photonic crystal fiber, ACS Photonics 5(6), 2018, pp. 2426-2430, DOI: 10.1021/acsphotonics.8b00197.

[2] Petersen C.R., Engelsholm R.D., Markos C., Brilland L., Caillaud C., Trolès J., Bang O., Increased mid-infrared supercontinuum bandwidth and average power by tapering large-mode-area chalcogenide photonic crystal fibers, Optics Express 25(13), 2017, pp. 15336-15348, DOI: 10.1364/ OE.25.015336.

[3] Herrmann J., Griebner U., Zhavoronkov N., Husakou A., Nickel D., Knight J.C., Wadsworth W.J., RusSell P.St.J., KoRn G., Experimental evidence for supercontinuum generation by fission of higher-order solitons in photonic fibers, Physical Review Letters 88(17), 2002, article 173901, DOI: 10.1103/PhysRevLett.88.173901.

[4] Fu L., Thomas B.K., Dong L., Efficient supercontinuum generations in silica suspended core fibers, Optics Express 16(24), 2008, pp. 19629-19642, DOI: 10.1364/OE.16.019629.

[5] MitschKe F.M., Mollenauer L.F., Discovery of the soliton self-frequency shift, Optics Letters 11(10), 1986, pp. 659-661, DOI: 10.1364/OL.11.000659.

[6] Zhao S., YANG H., ZhaO C., XIAO Y., Harnessing rogue wave for supercontinuum generation in cascaded photonic crystal fiber, Optics Express 25(7), 2017, pp. 7192-7202, DOI: 10.1364/OE.25.007192.

[7] Roy S., Bhadra S.K., Agrawal G.P., Effects of higher-order dispersion on resonant dispersive waves emitted by solitons, Optics Letters 34(13), 2009, pp. 2072-2074, DOI: 10.1364/OL.34.002072.

[8] Mahalingam A., Mani Rajan M.S., Influence of generalized external potentials on nonlinear tunneling of nonautonomous solitons: soliton management, Optical Fiber Technology 25, 2015, pp. 44-50, DOI: $10.1016 /$ j.yofte.2015.07.013.

[9] Feng X., Poletti F., Camerlingo A., Parmigiani F., Petropoulos P., Horak P., Ponzo G.M., Petrovich M., Shi J., Loh W.H., Richardson D.J., Dispersion controlled highly nonlinear fibers for all-optical processing at telecoms wavelengths, Optical Fiber Technology 16(6), 2010, pp. 378 -391, DOI: 10.1016/j.yofte.2010.09.014.

[10] Poletti F., Horak P., Richardson D.J., Soliton spectral tunneling in dispersion-controlled holey fibers, IEEE Photonics Technology Letters 20(16), 2008, pp. 1414-1416, DOI: 10.1109/LPT.2008.927881.

[11] Kibler B., Lacourt P.A., Courvoisier F., Dudley J.M., Soliton spectral tunnelling in photonic crystal fibre with sub-wavelength core defect, Electronics Letters 43(18), 2007, pp. 967-968, DOI: 10.1049/ el:20071553.

[12] Guo H., WANG S., Zeng X., BACHE M., Understanding soliton spectral tunneling as a spectral coupling effect, IEEE Photonics Technology Letters 25(19), 2013, pp. 1928-1931, DOI: 10.1109/LPT. 2013.2279122. 
[13] Napierala M., Nasilowski T., Bereś-Pawlik E., Mergo P., Berghmans F., Thienpont H., Large -mode-area photonic crystal fiber with double lattice constant structure and low bending loss, Optics Express 19(23), 2011, pp. 22628-22636, DOI: 10.1364/OE.19.022628.

[14] Stepniewski G., Klimczak M., Bookey H., Siwicki B., Pysz D., Stepien R., Kar A.K., Waddie A.J., TAGHIZADEH M.R., BUCZYNSKI R., Broadband supercontinuum generation in normal dispersion all -solid photonic crystal fiber pumped near $1300 \mathrm{~nm}$, Laser Physics Letters 11(5), 2014, article 055103, DOI: $10.1088 / 1612-2011 / 11 / 5 / 055103$.

[15] Knight J.C., Photonic crystal fibres, Nature 424, 2003, pp. 847-851, DOI: 10.1038/nature01940.

[16] Stark S.P., Biancalana F., Podlipensky A., Russell P.St.J., Nonlinear wavelength conversion in photonic crystal fibers with three zero-dispersion points, Physical Review A 83(2), 2011, article 023808, DOI: 10.1103/PhysRevA.83.023808.

[17] Akhmediev N., Karlsson M., Cherenkov radiation emitted by solitons in optical fibers, Physical Review A 51(3), 1995, pp. 2602-2609, DOI: 10.1103/PhysRevA.51.2602.

[18] Kaup D.J., Newell A.C., An exact solution for a derivative nonlinear Schrödinger equation, Journal of Mathematical Physics 19(4), 1978, pp. 798-801, DOI: 10.1063/1.523737.

[19] Wang S., Guo H., FAn D., Bai X., Zeng X., Analysis of cascaded soliton spectral tunneling effect in segmented fibers with engineered dispersion, IEEE Photonics Journal 5(6), 2013, article 6100608, DOI: 10.1109/JPHOT.2013.2290001.

[20] Marest T., Braud F., Conforti M., Wabnitz S., Mussot A., Kudlinski A., Longitudinal soliton tunneling in optical fiber, Optics Letters 42(12), 2017, pp. 2350-2353, DOI: 10.1364/OL.42.002350.

[21] Zhao S., Yang H., Zhao Y., Xiao Y., Supercontinuum manipulation based on the influence of chirp on soliton spectral tunneling, Chinese Physics B 27(11), 2018, article 114219, DOI: 10.1088/1674-1056/ 27/11/114219.

[22] Agrawal G.P., Nonlinear Fiber Optics, 4th Ed., New York 2007.

[23] Joly N.Y., Omenetto F.G., Efimov A., Taylor A.J., Knight J.C., Russell P.St.J., Competition between spectral splitting and Raman frequency shift in negative-dispersion slope photonic crystal fiber, Optics Communications 248(1-3), 2005, pp. 281-285, DOI: 10.1016/j.optcom.2004.11.091.

[24] Yang H., Han F., Hu H., Wang W., Zeng Q., Spectral-temporal analysis of dispersive wave generation in photonic crystal fibers of different dispersion slope, Journal of Modern Optics 61(5), 2014, pp. 409-414, DOI: 10.1080/09500340.2014.890252.

[25] Zhao S., Yang H., Chen N., Fu X., Zhao C., Soliton trapping of dispersive waves in photonic crystal fiber with three zero-dispersion wavelengths, IEEE Photonics Journal 7(5), 2015, article 7102709, DOI: $10.1109 / \mathrm{JPHOT} .2015 .2486683$.

Received December 7, 2019

in revised form February 19, 2020 